\title{
Alterations in oestrogen metabolism: implications for higher penetrance of familial pulmonary arterial hypertension in females
}

\author{
E.D. Austin*, J.D. Cogan*, J.D. West" , L.K. Hedges*, R. Hamid*, E.P. Dawson \\ L.A. Wheeler" ${ }^{\#}$ F.F. Parl ${ }^{\mp}$, J.E. Loyd ${ }^{\#}$ and J.A. Phillips III*
}

ABSTRACT: Mutations in bone morphogenetic protein receptor type 2 (BMPR2) cause familial pulmonary arterial hypertension (FPAH), but the penetrance is reduced and females are significantly overrepresented. In addition, gene expression data implicating the oestrogenmetabolising enzyme CYP1B1 suggests a detrimental role of oestrogens or oestrogen metabolites. We examined genetic and metabolic markers of altered oestrogen metabolism in subjects with a BMPR2 mutation.

Genotypes for CYP1B1 Asn453Ser (N453S) were determined for 140 BMPR2 mutation carriers (86 females and 54 males). Nested from those subjects, a case-control study of urinary oestrogen metabolite levels (2-hydroxyoestrogen (2-OHE) and 16a-hydroxyoestrone (16a-OHE 1 )) was conducted in females (five affected mutation carriers versus six unaffected mutation carriers).

Among females, there was four-fold higher penetrance among subjects homozygous for the wild-type genotype $(N / N)$ than those with $N / S$ or $S / S$ genotypes $(p=0.005)$. Consistent with this finding, the 2-OHE/16 $\alpha-\mathrm{OHE}_{1}$ ratio was 2.3-fold lower in affected mutation carriers compared to unaffected mutation carriers $(p=0.006)$.

Our findings suggest that variations in oestrogens and oestrogen metabolism modify FPAH risk. Further investigation of the role of oestrogens in this disease with profound sex bias may yield new insights and, perhaps, therapeutic interventions.

KEYWORDS: Bone morphogenetic protein receptor 2, CYP1B1, genetic polymorphism, oestrogen, pulmonary hypertension, sex

amilial pulmonary arterial hypertension (FPAH) is caused, in $80 \%$ of families, by autosomal dominant mutations in the gene encoding bone morphogenetic protein receptor type 2 (BMPR2). However, it is unclear why $B M P R 2$ mutations have reduced penetrance, and why, after puberty, females with mutations are about 2.5-fold more likely to develop FPAH than males [1]. The excess of adult females suggests oestrogen as a possible disease modifier, as does a lower prevalence among pre-pubertal females compared with males $[2,3]$. A detrimental role for oestrogens in FPAH is supported by studies that show oestrogen is a potent mitogen of pulmonary vascular smooth muscle cells [4].

Recently, our studies of BMPR2 mutation carriers implicated altered oestrogen metabolism as a key factor in the penetrance of FPAH in females.
Using genome expression arrays confirmed by RT-PCR, we found significantly decreased transcript levels of cytochrome P450 1B1 (CYP1B1; a cytochrome p450 family enzyme critical to oestrogen metabolism) in affected female compared with unaffected female BMPR2 mutation carriers [5]. CYP1B1 is highly expressed in the lung, and it is a modifier for several cancers, including lung and breast cancers [6-14]. CYP1B1 catalyses the oxidation of oestrogens to 2hydroxy (2-OHE) and 4-hydroxy (4-OHE) oestrogens, and metabolises environmental toxins, including tobacco smoke [15].

Oxidation of oestrogens also occurs by hydroxylation at the $\mathrm{C}-16$ position by other $\mathrm{P} 450$ enzymes, predominantly resulting in $16 \alpha$-hydroxyoestrone $\left(16 \alpha-\mathrm{OHE}_{1}\right)$ in extrahepatic tissues $[16,17]$. Data suggest that, unlike the weak
AFFILIATIONS

Depts of *Pediatrics,

\#Medicine, and

"Pathology, Vanderbilt University

Medical Center, Nashville, TN, USA

CORRESPONDENCE

E.D. Austin

Dept of Pediatrics,

Division of Pulmonary, Allergy,

and Immunology Medicine,

DD-2205 Medical Center North

Vanderbilt University School of

Medicine

Nashville

TN

37232-2578

USA

E-mail: eric.austin@vanderbilt.edu

Received:

Jan 212009

Accepted after revision:

March 232009

First published online:

April 082009

European Respiratory Journal Print ISSN 0903-1936

Online ISSN 1399-3003 
mitogen 2-OHE, $16 \alpha-\mathrm{OHE}_{1}$ stimulates cellular proliferation by constitutively activating the oestrogen receptor. In addition to being more mitogenic than $2-\mathrm{OHE}, 16 \alpha-\mathrm{OHE}_{1}$ may also be more genotoxic. Thus, individuals who metabolise a larger proportion of oestrogen to $16 \alpha-\mathrm{OHE}_{1}$ may be at increased risk of diseases that result from both the mitogenic and genotoxic effects of oestrogens, such as breast and prostate cancers [18].

We hypothesised that oestrogens may contribute to FPAH, and that the risk may be altered by variations in CYP1B1. To test this, we studied the relationship between the CYP1B1 Asn453Ser (N453S) genetic polymorphism and FPAH penetrance in BMPR2 mutation carriers [19]. We also determined the urinary $2-\mathrm{OHE} / 16 \alpha-\mathrm{OHE}_{1}$ ratio, one biological measure of CYP1B1 activity, among a group of females nested from our polymorphism study. Our genetic and biochemical results suggest that imbalanced oestrogen metabolism may contribute to the pathogenesis of FPAH, and that further analysis of this pathway may lead to new biological markers and therapeutic treatments for all forms of $\mathrm{PAH}$.

\section{MATERIALS AND METHODS}

\section{Study population}

The Vanderbilt pulmonary arterial hypertension (PAH) research registry provided the study base from which the subjects in this study were recruited. The registry contains participants with idiopathic $\mathrm{PAH}$, as well as participants affected with FPAH, bloodline relatives of those affected and unrelated family members of all ages. Study subjects for the registry, the majority of whom are now deceased, were recruited via the Vanderbilt Pulmonary Hypertension Center (Nashville, TN, USA), the Pulmonary Hypertension Association (Silver Spring, MD, USA) and the National Institutes of Health (Bethesda, MD, USA) clinical trials website (http://clinicaltrials.gov). BMPR2 mutations were detected in a large proportion of families with FPAH followed by the registry. These mutations were heterogeneous and included nonsense, missense and frameshift alterations, as well as insertion-deletion mutations that lead to splicing errors. In addition the detected BMPR2 mutations were heterogeneous in location across the gene.

For this study, subjects diagnosed with FPAH who had a $B M P R 2$ mutation were considered affected BMPR2 mutation carriers (AMCs). Subjects who have never been diagnosed with FPAH but have a BMPR2 mutation were considered unaffected BMPR2 mutation carriers (UMCs). Clinical information on AMCs, including cardiac catheterisation data and therapeutic regimens, was available from medical history and records. Specialist physicians in their geographic regions identified subjects as affected with FPAH, and our investigators reviewed all medical records for accuracy of diagnosis. We defined FPAH diagnostically, either by autopsy results showing plexogenic pulmonary arteriopathy in the absence of alternative causes, such as congenital heart disease, or by clinical and cardiac catheterisation criteria. These criteria included a mean pulmonary arterial pressure $>25 \mathrm{mmHg}$ with a pulmonary capillary or left atrial pressure $<15 \mathrm{mmHg}$, and exclusion of other causes of pulmonary hypertension, in accordance with accepted international standards of diagnostic criteria [20]. All subjects designated as UMCs were seen by a physician within 1 yr of sample acquisition, and without symptoms or signs of PAH at time of sample acquisition.

The Vanderbilt University Medical Center (Nashville, TN, USA) institutional review board approved all study protocols. All participants gave informed written consent to participate in genetic and clinical studies. Samples were obtained at the time of clinic visit or hospitalisation, or by post via a kit for collection of whole blood and DNA.

For our CYP1B1 N453S polymorphism study, 140 BMPR2 mutation carriers were recruited, including 86 females and 54 males. Relevant clinical characteristics for these subjects are listed in table 1. Subsequently, a nested case-control cohort study of urinary oestrogen metabolites was performed in 11 female BMPR2 mutation carriers. This cohort was composed of five female patients previously diagnosed with FPAH (AMCs) and six females who were UMCs. Relevant clinical characteristics for the AMCs included in this nested cohort are listed in table 2. The following criteria had to be met for inclusion in the nested case-control cohort study: 1) living; 2) female; 3) not currently pregnant; 4) not pregnant within the past $1 \mathrm{yr}$; 5) not hysterectomised or with an oophorectomy; and 6) not currently taking exogenous steroid hormones. UMCs were not eligible if taking prescribed or over-the-counter medications or supplements.

Overnight 12-h urine void collections were obtained from all participants following previously established protocols. Urine samples from pre-menopausal females were obtained in the mid-luteal phase of the menstrual cycle (4-6 days prior to the estimated start of the next menses) and subsequent menses confirmed. Urine was stored at $4^{\circ} \mathrm{C}$ on ice or in a refrigerator until processing for storage. To prevent oxidation of metabolites during storage, $50 \mathrm{mg}$ of ascorbic acid was added to $50 \mathrm{~mL}$ of urine $\left(1 \mathrm{mg} \cdot \mathrm{mL}^{-1}\right)$ and the sample mixed thoroughly. Samples were aliquoted into conical tubes and stored at $-80^{\circ} \mathrm{C}$ within $24 \mathrm{~h}$ of collection.

\begin{tabular}{|c|c|c|c|}
\hline \multirow[t]{2}{*}{ TABLE 1} & \multicolumn{3}{|c|}{$\begin{array}{l}\text { Clinical characteristics of the } 140 \text { BMPR2 } \\
\text { mutation carriers genotyped for the CYP1B1 } \\
\text { Asn453Ser ( } 453 \mathrm{~N} / \mathrm{S}) \text { polymorphism }\end{array}$} \\
\hline & & AMCs & UMCs \\
\hline \multicolumn{2}{|l|}{ Subjects $\mathbf{n}$} & 92 & 48 \\
\hline \multicolumn{2}{|l|}{ Females } & $62(67.4)$ & $24(50.0)$ \\
\hline \multicolumn{2}{|c|}{ Diagnosis age yrs } & $35.9 \pm 13.7$ & NA \\
\hline \multicolumn{4}{|c|}{ Baseline haemodynamic data at diagnosis } \\
\hline \multicolumn{2}{|c|}{ Right atrial pressure $\mathrm{mmHg}$} & $10.9 \pm 8.4$ & NA \\
\hline \multicolumn{2}{|l|}{$\bar{P}$ pa $\mathrm{mmHg}$} & $58.3 \pm 13.2$ & NA \\
\hline \multicolumn{2}{|c|}{ Cardiac index $\mathrm{L} \cdot \mathrm{min} \cdot \mathrm{m}^{-2}$} & $1.87 \pm 0.7$ & NA \\
\hline \multicolumn{2}{|c|}{ Indexed PVR U. $\mathrm{m}^{2}$} & $18.3 \pm 14.0$ & NA \\
\hline \multicolumn{4}{|c|}{$\begin{array}{l}\text { Data are presented as } n(\%) \text { or mean } \pm S D \text {, unless otherwise stated. AMC: } \\
\text { affected mutation carrier; UMC: unaffected mutation carrier; } \bar{P} \text { pa: mean } \\
\text { pulmonary artery pressure; PVR: pulmonary vascular resistance; NA: not } \\
\text { available. }\end{array}$} \\
\hline
\end{tabular}




\begin{tabular}{|c|c|c|c|c|c|c|}
\hline \multirow[t]{2}{*}{ TABLE 2} & \multicolumn{6}{|c|}{$\begin{array}{l}\text { Clinical characteristics of the five affected BMPR2 mutation carriers (AMCs) studied in the nested case-control cohort } \\
\text { study }\end{array}$} \\
\hline & & AMC 1 & AMC 2 & AMC 3 & AMC 4 & AMC 5 \\
\hline \multicolumn{2}{|c|}{ Diagnosis age yrs } & 31 & 36 & 64 & 53 & 43 \\
\hline \multicolumn{2}{|c|}{ Current age yrs } & 31 & 52 & 74 & 57 & 48 \\
\hline \multicolumn{2}{|c|}{ Current BMI $\mathrm{kg} \cdot \mathrm{m}^{-2}$} & 21.6 & 22.0 & 19.4 & 29.9 & 23.9 \\
\hline \multicolumn{2}{|c|}{ Pregnancies $n$} & 3 & 3 & 11 & 1 & 2 \\
\hline \multicolumn{2}{|c|}{ Offspring $n$} & 3 & 3 & 7 & 1 & 2 \\
\hline \multicolumn{2}{|c|}{ Menopause status } & Pre & Post & Post & Post & Pre \\
\hline \multicolumn{2}{|c|}{ OCP or HRT at diagnosis } & No & Yes & Yes & No & No \\
\hline \multicolumn{2}{|c|}{ NYHA functional class at diagnosis } & 3 & 2 & 2 & 2 & 3 \\
\hline \multicolumn{7}{|c|}{ Baseline haemodynamic data at diagnosis } \\
\hline \multicolumn{2}{|c|}{ Responsive to acute vasodilator challenge } & No & No & No & No & No \\
\hline \multicolumn{7}{|c|}{ PAH-specific therapies } \\
\hline \multicolumn{2}{|c|}{ Prostanoids } & Yes & Yes & Yes & Yes & No \\
\hline \multicolumn{2}{|c|}{ Phosphodiesterase- 5 inhibitors } & Yes & No & Yes & No & No \\
\hline \multicolumn{2}{|c|}{ Endothelin receptor antagonists } & No & No & No & Yes & Yes \\
\hline
\end{tabular}

BMI: body mass index; OCP: oral contraceptive pill; HRT: hormone replacement therapy; NYHA: New York Heart Association; $\bar{P}$ pa: mean pulmonary artery pressure; PVR: pulmonary vascular resistance; PAH: pulmonary arterial hypertension.

\section{Laboratory analyses}

Genomic DNA was isolated from whole blood using Puregene $^{\circledR}$ DNA Purification Kit (Gentra, Minneapolis, MN, USA) according to the manufacturer's protocol for genotyping. As previously described, established lymphoblastoid cell lines of participants were grown in RPMI-1640 containing 15\% fetal bovine serum, penicillin $100 \mu \mathrm{g} \cdot \mathrm{mL}^{-1}$ and streptomycin $100 \mu \mathrm{g} \cdot \mathrm{mL}^{-1}$ in T-25 flasks to provide RNA. Cells were grown to $\sim 1 \times 10^{6}$ cells per flask and incubated in the presence or absence of puromycin $\left(100 \mu \mathrm{g} \cdot \mathrm{mL}^{-1}\right)$ for $16 \mathrm{~h}$ before harvesting. Cells were harvested using RNAeasy Mini Kit including the optional DNase treatment (Qiagen Inc., Valencia, CA, USA) [21]. We performed BMPR2 gene mutation detection by sequencing exons and exon intron boundaries of genomic DNA and RT-PCR analysis as previously described [21, 22]. All detected BMPR2 mutations in this study have been previously reported, and are included in a recent summary of detectable BMPR2 mutations [23].

Genotyping of the samples for the CYP1B1 N453S genetic polymorphism (rs1800440) was performed using the TaqMan SNP Assay, C_11642651_30 from Applied Biosystems (Foster City, CA, USA). The samples were amplified with Universal Master Mix (Applied Biosystems) and run and analysed on the 7500 Real Time PCR System (Applied Biosystems) using allelic discrimination cycling conditions. Sample genotyping was performed in triplicate by staff blinded to the subjects' phenotype; nontemplate controls were also included. The accuracy of genotyping data was tested by direct DNA sequencing of $\sim 15 \%$ of samples analysed, and all results were in $100 \%$ concordance.
All urinary oestrogen metabolite study samples were run together in a blinded manner. The samples were thawed at room temperature, centrifuged at $2000 \times g$ for $10 \mathrm{~min}$, and $10 \mathrm{~mL}$ of clear urine was extracted for each assay. Urinary oestrogen metabolites were quantitated using a monoclonal antibody-based enzyme assay (Estramet $2 / 16^{\mathrm{TM}}$; Immuna Care, Blue Bell, PA, USA). This assay was previously validated by gas chromatography-mass spectroscopy methods and found to reliably measure $2-\mathrm{OHE}$ and $16 \alpha-\mathrm{OHE}_{1}$ levels [24-26]. Urine samples from pre-menopausal females were pre-diluted 1:4 using the manufacturer's diluent and assayed according to the manufacturer's protocol. All urine samples were run in sextuplet, with controls, and standards in triplicate. Samples, controls and standards were pipetted into individual microtubes in sextuplet, with $190 \mu \mathrm{L}$ hydrolysis buffer added to each tube followed by a 2 -h covered incubation at room temperature. Neutralisation buffer $(200 \mu \mathrm{L}$ per well) was added to the enzyme digest to bring the sample to a $\mathrm{pH}$ of 7.0 , and $75 \mu \mathrm{L}$ of each sample was added to two 96-well microtitre plates (one for the determination of 2-OHE and one for $16 \alpha-\mathrm{OHE}_{1}$ ) coated with polyclonal rabbit anti-mouse immunoglobulin. Immediately prior to use, the plates were activated by washing with buffer using an automatic plate washer (Bio-Rad model 1575 Immunowash; Bio-Rad Laboratories Inc., Hercules, CA, USA) according to the manufacturer's package insert. To each respective assay plate was added $75 \mu \mathrm{L}$ of a conjugate buffer solution containing metabolite-specific 2-OHE:alkaline phosphatase or $16 \alpha-$ $\mathrm{OHE}_{1}$ :alkaline phosphatase. The plates were sealed and incubated for $3 \mathrm{~h}$ at room temperature, then washed with an automatic plate washer according to the manufacturer's insert. 
Stabilised paranitrophenyl phosphate $(100 \mu \mathrm{L})$ was added to each well, and the enzyme product (a colour dye) was inversely proportional to the concentration of free antigen (oestrogen metabolite).

Absorbance was read kinetically by end-point analysis with a Bio-Rad iMark Microplate Absorbance Reader (Bio-Rad Laboratories Inc.) at $405 \mathrm{~nm} 60 \mathrm{~min}$ after adding the paranitrophenyl phosphate solution. The data were reduced using Microplate Manager 6 Application Software (Bio-Rad Laboratories Inc.). A four-parameter fit curve of the log of the concentration versus absorbance at $405 \mathrm{~nm}$ yielded a sigmoidal standard curve with a linear range of $1-15 \mathrm{ng} \cdot \mathrm{mL}^{-1}$ of urine. Urinary creatinine was quantitated in duplicate using a commercially available colorimetric assay (Ortho-Clinical Diagnostics, Rochester, NY, USA) and was used to normalise oestrogen metabolite levels. Results for the concentration of each metabolite were reported as nanogrammes per millilitre per milligram of creatinine. Samples from each patient were assayed together in a single assay to eliminate inter-assay variation.

\section{Statistical analysis}

We used the Chi-squared test to determine if the CYP1B1 genotypes were in Hardy-Weinberg equilibrium and to determine the $\mathrm{p}$-values for differences observed in genotype frequencies between AMCs and UMCs. Associations between CYP1B1 genotypes and FPAH penetrance were examined by conditional logistic regression to calculate odds ratios and $95 \%$ confidence intervals. Because of sample size considerations, we combined data from heterozygotes (N/S) and homozygotes (S/S) for statistical analyses. Detailed pedigree analysis showed that subjects were too distantly related to justify the use of statistical methods to evaluate association between genetic markers and disease status using family-based data.

All urinary oestrogen metabolite laboratory experiments were performed in sextuplet, and the mean value recorded for each subject. To account for any non-normal data distributions, and for consistency of analysis, statistical significance was evaluated using nonparametric statistical techniques with two-sided

\begin{tabular}{|c|c|c|c|}
\hline \multirow[t]{2}{*}{ TABLE 3} & \multicolumn{3}{|c|}{$\begin{array}{l}\text { Distribution of the CYP1B1 Asn453Ser ( } 453 \mathrm{~N} / \mathrm{S} \text { ) } \\
\text { genotypes according to familial pulmonary } \\
\text { arterial hypertension phenotype: affected } \\
\text { (AMCs) and unaffected BMPR2 mutation carriers } \\
\text { (UMCs) }\end{array}$} \\
\hline & AMC & UMC & $p$-value \\
\hline \multicolumn{4}{|l|}{ Females } \\
\hline $\mathrm{N} / \mathrm{N}$ & $46(74)$ & $10(42)$ & 0.005 \\
\hline $\mathrm{N} / \mathrm{S}$ or $\mathrm{S} / \mathrm{S}$ & $16(26)$ & $14(58)$ & \\
\hline Total & $62(100)$ & $24(100)$ & \\
\hline \multicolumn{4}{|l|}{ Males } \\
\hline $\mathrm{N} / \mathrm{N}$ & $18(60)$ & $17(71)$ & 0.407 \\
\hline$N / S$ or $S / S$ & $12(40)$ & 7 (29) & \\
\hline Total & $30(100)$ & $24(100)$ & \\
\hline
\end{tabular}

Data are presented as n (\%), unless otherwise stated. testing, with a p-value of $<0.05$ considered statistically significant. Urinary oestrogen metabolite levels were compared between the AMC and UMC groups using the nonparametric Mann-Whitney U-test (Wilcoxon rank-sum test). Oestrogen metabolite values are expressed as mean $\pm \mathrm{SD}$ or median (interquartile range).

All p-values are two-sided. Statistical analysis was performed using the statistical package SPSS for Windows (Version 16.0; SPSS Inc., Chicago, IL, USA).

\section{RESULTS}

The distributions of the CYP1B1 N453S genotypes among the 140 subjects tested were compared between AMCs $(n=92)$ and UMCs $(n=48)$ according to sex. Among females $(n=86)$, the genotype frequencies from $\operatorname{AMCs}(n=62)$ were significantly different from the UMCs $(n=24)$ (Chi-squared 8.06, degrees of freedom (df) $1, p=0.005)$, with a greater penetrance among subjects homozygous for the wild-type genotype $(\mathrm{N} / \mathrm{N})$ (table 3). Compared with the $\mathrm{N} / \mathrm{N}$ genotype, the unadjusted odds ratio for the group with N/S or S/S genotypes was 0.248 (95\% CI 0.092-0.669), indicating a four-fold higher penetrance among female $\mathrm{N} / \mathrm{N}$ homozygotes compared with those with $\mathrm{N} / \mathrm{S}$ or S/S genotypes. There was no difference among males $(\mathrm{n}=54)$ (Chi-squared 0.69, df $1, \mathrm{p}=0.407)$ between AMCs and UMCs.

The nested case-control study evaluated urinary metabolites in 11 nonsmoking females from the genotype study. The five AMCs (age $52.4 \pm 15.3 \mathrm{yrs}$ ) had a mean body mass index (BMI) of $23.7 \mathrm{~kg} \cdot \mathrm{m}^{-2}$, and three out of five were post-menopausal. The six UMCs (age 57.3 \pm 10.8 yrs) were healthy nonsmokers with no known diseases and taking no medications, with a mean BMI of $25.4 \mathrm{~kg} \cdot \mathrm{m}^{-2}$, and five out of six were postmenopausal.

Cases (AMCs) and controls (UMCs) were not statistically different in terms of age $(p=0.463)$, weight and height (BMI; $p=0.268$ ), age at menarche, number of pregnancies, parity or menstrual status. Urine creatinine levels were not statistically different between cases and controls $\left(0.84\right.$ versus $1.16 \mathrm{mg} \cdot \mathrm{mL}^{-1}$; $\mathrm{p}=0.361)$

There were no statistically significant differences in oestrogen metabolite concentrations, although the sum of urinary metabolites $\left(2-\mathrm{OHE}\right.$ and $\left.16 \alpha-\mathrm{OHE}_{1}\right)$ measured was higher in the AMCs than UMCs, as was the measured level of $16 \alpha-\mathrm{OHE}_{1}$. Importantly, the $2-\mathrm{OHE} / 16 \alpha-\mathrm{OHE}_{1}$ ratio was 2.3 -fold lower in AMCs compared with UMCs (0.65 versus $1.48 \mathrm{ng}$ per mg creatinine per $\mathrm{mL} ; \mathrm{p}=0.006)$, with a distinct separation in the ranges of values between the two groups (table 4 and fig. 1). These findings were unchanged by the stratification into postmenopausal subjects $(0.57(n=3)$ versus $1.47(n=4)$ ng per $\mathrm{mg}$ creatinine per $\mathrm{mL} ; \mathrm{p}=0.034)$ and pre-menopausal subjects $(0.76$ $(\mathrm{n}=2)$ versus $1.83(\mathrm{n}=1)$ ng per $\mathrm{mg}$ creatinine per $\mathrm{mL} ; \mathrm{p}=0.221)$.

\section{DISCUSSION}

The 2.5-fold female to male disparity in penetrance, and our recent discovery that transcript levels of a major oestrogenmetabolising enzyme (CYP1B1) may modify disease risk in females, suggests that oestrogens or oestrogen metabolites may influence PAH risk in BMPR2 mutation carriers [5]. To pursue this further, we determined the genotype of a CYP1B1 


\begin{tabular}{|c|c|c|c|c|}
\hline \multirow{2}{*}{$\begin{array}{l}\text { TABLE } 4 \\
\text { Urine metab }\end{array}$} & \multicolumn{4}{|c|}{$\begin{array}{l}\text { Urinary oestrogen metabolite values in affected } \\
\text { BMPR2 mutation carriers (AMCs) versus } \\
\text { unaffected BMPR2 mutation carriers (UMCs) }\end{array}$} \\
\hline & lites & $\mathrm{AMC}^{\#}$ & UMC" & $p$-value ${ }^{+}$ \\
\hline \multicolumn{2}{|l|}{ 2-OHE } & $6.96 \pm 9.3$ & $6.27 \pm 1.2$ & 0.201 \\
\hline \multicolumn{2}{|l|}{$16 \alpha-\mathrm{OHE}_{1}$} & $10.05 \pm 13.4$ & $4.30 \pm 0.9$ & 0.715 \\
\hline \multirow{2}{*}{\multicolumn{2}{|c|}{ Sum of $2-\mathrm{OHE}$ and $16 \alpha-\mathrm{OHE}_{1}$}} & $17.01 \pm 22.6$ & $10.57 \pm 1.8$ & 0.361 \\
\hline & & $0.65 \pm 0.3$ & $1.48 \pm 0.2$ & 0.006 \\
\hline
\end{tabular}

polymorphism previously implicated in breast, endometrial and prostate cancers, and also measured oestrogen metabolites in AMCs and UMCs. We found that AMCs are more likely to be CYP1B1 N/N than UMCs, and to have lower 2-OHE/16 $\alpha$ $\mathrm{OHE}_{1}$ ratios in urinary oestrogen metabolites consistent with diminished CYP1B1 activity. Our results suggest that oestrogen or oestrogen metabolites participate in the pathogenesis of FPAH, and provide an important clue toward understanding the mechanisms behind sex differences in disease penetrance.

While FPAH is an autosomal dominant disease, the female predominance ( $\sim 2.5$ females to one male), reduced penetrance and variable age of diagnosis strongly suggest that combinations of modifying exposures may interact to promote its pathogenesis [27]. However, sex remains the most definitive modifier of disease, suggesting that oestrogens may be important. Additional features that also implicate oestrogens include the association of pharmacological oestrogen exposures with PAH onset and the onset of PAH around pregnancy [2]. Furthermore, data from our research registry also demonstrates that fewer of the female diagnoses and deaths occur during childhood compared with males (13\% of females versus $21 \%$ of males, $\mathrm{p}=0.031$ for difference prior to age $18 \mathrm{yrs}$; fig. 2), but the reverse for adults. The smaller difference between adult female and male groups likely reflects the importance of additional factors in the pathophysiology of disease as a BMPR2 mutation carrier ages.

Experimental data concerning the role of oestrogens in pulmonary vascular disease implicate oestrogens as promoters of inappropriate vascular proliferation and cell damage, but also as inhibitors of pulmonary vasoreactivity. In vitro, oestrogen is mitogenic and promotes proliferation of pulmonary vascular smooth muscle cells, while the anti-oestrogen drug tamoxifen blocks this effect [4]. In chronic hypoxia models oestrogen attenuates pulmonary artery vasoreactivity and acute hypoxic pulmonary vasoconstriction [28]. The implication of oestrogens in the pathogenesis of BMPR2associated FPAH is consistent with hypotheses that PAH is primarily driven by pro-proliferative and anti-apoptotic forces within the pulmonary vascular bed [29].

The CYP multigene family of enzymes metabolise many endogenous and exogenous compounds [30]. CYP1B1 is a key enzyme in oestrogen metabolism associated with several hormone-regulated cancers, including breast, endometrial and

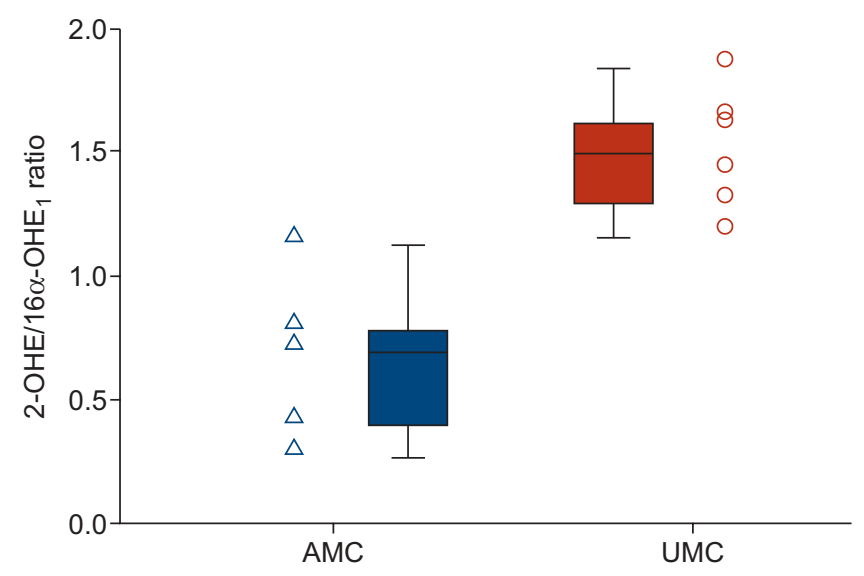

FIGURE 1. Ratio of urinary 2-hydroxyoestrogen (2-OHE) to $16 \alpha$-hydroxyoestrone $\left(16 \alpha-\mathrm{OHE}_{1}\right)$ in affected BMPR2 mutation carriers (AMCs) versus unaffected BMPR2 mutation carriers (UMCs) (ng per mg creatinine per $\mathrm{mL}$ ). AMCs have a significantly reduced ratio compared to UMCs $(p=0.006)$. Data points and box plots are shown for each group. Box and whisker plots represent the median value, interquartile range and highest and lowest values.

prostate. It is an active extrahepatic enzyme that is highly expressed in oestrogen-regulated tissues, such as the breast, but also in the lung, suggesting that CYP1B1 is important in the localised metabolic control of oestrogen balance [7]. Interestingly, CYP1B1 is transcriptionally activated by oestrogens and oestrogen metabolites, via the oestrogen receptor, to hydroxylate oestrogens into the 2-OHE and 4-OHE metabolites [31]. Thus, oestrogens are not only substrates, but activators of CYP1B1 activity by direct interaction between the oestrogen receptor and oestrogen response elements on the CYP1B1 gene.

Oestrogens and most oestrogen metabolites are pro-proliferative. However, not all oestrogen metabolites are equal in their effects. In particular, $4-\mathrm{OHE}$ and $16 \alpha-\mathrm{OHE}_{1}$ exert far greater mitogenic and genotoxic effects than $2-\mathrm{OHE}$, making the ratio of $2-\mathrm{OHE}$ to $4-\mathrm{OHE}$ and/or $16 \alpha-\mathrm{OHE}_{1}$ important in the genesis of hormonal-based cancers [32-36]. For example, it has been hypothesised that metabolism favouring 2-OHE over the $16 \alpha-$ $\mathrm{OHE}_{1}$ pathway may be protective of breast cancer in at-risk subjects $[18,37]$. The substantially lower ratio of $2-\mathrm{OHE} / 16 \alpha-$ $\mathrm{OHE}_{1}$ we observed in AMCs compared with UMCs is consistent with the expectation that a lower ratio is detrimental to those subjects already at increased genetic risk of developing PAH.

Consistent with this finding, 2-methoxyoestradiol and its synthetic analogue 2-ethoxyoestradiol possess potent antimitogenic properties. These compounds, similar to 2-OHE, are metabolites of oestrogen without oestrogenic effects that may antagonise oestrogen. In fact, in a rat model of monocrotaline-induced pulmonary hypertension, treatment with both 2-methoxyoestradiol and 2-ethoxyoestradiol prevented pulmonary hypertension exacerbation and reduced mortality via attenuation of the inflammatory response and vascular remodelling $[38,39]$. The findings in our study would support the conclusion than certain non-oestrogenic metabolites of oestrogen may be protective, and perhaps of therapeutic benefit, in pulmonary hypertension. 


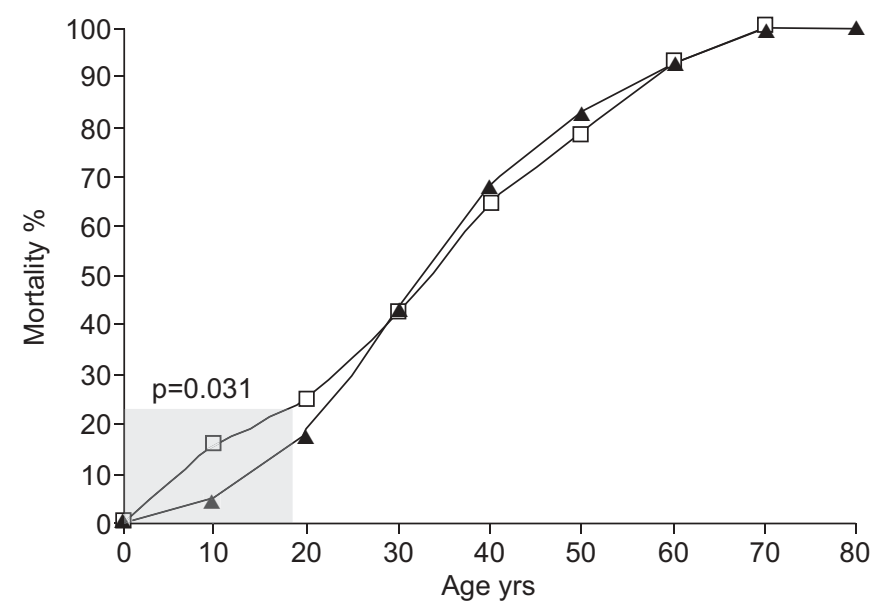

FIGURE 2. Cumulative mortality curve for all affected BMPR2 mutation carriers in the Vanderbilt familial pulmonary arterial hypertension research registry, represented as the percentage of all subjects by $\operatorname{sex}(\mathbf{\Lambda}$ : females; $\square$ : males) deceased at a given age. There is a statistically significant difference in the per cent mortality by age between the two groups prior to age 18 yrs (shaded; $p=0.031$ ). The difference in per cent mortality between males and females prior to age $18 \mathrm{yrs}$ reflects a lower prevalence among females in childhood compared with males.

While the present study is the first, to our knowledge, to suggest the possible contribution of CYP1B1 N453S genotypes and oestrogen metabolites to $\mathrm{PAH}$, our results are consistent with those of oncology researchers who have implicated this polymorphism in several oestrogen-regulated cancers. For example, studying individuals at risk of familial breast cancer, GREENLEE et al. [40] found $40 \%$ higher $2-\mathrm{OHE} / 16 \alpha-\mathrm{OHE}_{1}$ ratios in subjects heterozygous (N/S) or homozygous (S/S) compared with those with the homozygous $(\mathrm{N} / \mathrm{N})$ genotype. Our findings of increased penetrance (higher percentage of AMCs) among N/N homozygotes, as well as lower $2-\mathrm{OHE} / 16 \alpha-\mathrm{OHE}_{1}$ ratios in AMCs compared with UMCs, suggest that variations in oestrogen metabolism due to CYP1B1 genetic variation may influence disease in FPAH as well.

Our study has several strengths, including the availability of specimens from one of the largest registries of FPAH in the world and standardised methods of sample collection. However, several limitations in our study warrant caution for interpreting its findings, and suggest the importance of further investigation. First, within the limits of exclusion by family history, the majority of subjects included in our analysis are unrelated. While a small percentage are distantly related to one another, this population of subjects did not meet recommended criteria for the use of family-based tests of genetic analysis, which may have improved power to detect differences by genotypes [41]. Secondly, our hypothesis that oestrogens and oestrogen metabolites are important in $\mathrm{PAH}$ makes it likely that additional variations in CYP1B1 and/or other enzymes involved in oestrogen metabolism may also be important. This is particularly relevant because the formation and metabolism of oestrogen is a complicated process, involving multiple enzymes in addition to CYP1B1, including aromatase, CYP1A1, CYP3A4, and catechol-O-methyltransferase. In fact, pulmonary hypertension associated with liver disease, which also predominantly occurs in females, has recently been linked to alterations in oestrogen activity associated with polymorphisms in the gene that encodes the aromatase enzyme [42]. Thirdly, although we attempted to recruit all AMC and UMC members of our PAH research registry who met study criteria, and the number of subjects available for this study represents a significant fraction of all known FPAH cases in North America, the number remains small. Although the large $2-\mathrm{OHE} / 16 \alpha-\mathrm{OHE}_{1}$ ratio difference we observed between AMCs and UMCs was statistically significant despite the small number of subjects, we need to expand our study to include more subjects. Finally, we studied oestrogen metabolites previously reported to be important in other diseases with sex disparity, but did not measure other potentially important oestrogen metabolites which may impact risk.

In conclusion, our results support the hypothesis that variations in oestrogen metabolism may play a detrimental role in the development of FPAH, and that the risk of FPAH is influenced by variations in CYP1B1. Female BMPR2 mutation carriers with the $\mathrm{N} / \mathrm{N}$ genotype for CYP1B1 N453S have greater penetrance of $\mathrm{FPAH}$, consistent with prior evidence that this genotype is associated with a lower $2-\mathrm{OHE} / 16 \alpha-\mathrm{OHE}_{1}$ ratio and, thus, more mitogenic and genotoxic activity. As expected, female BMPR2 AMCs have statistically lower 2-OH/ $16 \alpha-\mathrm{OHE}_{1}$ ratios than female BMPR2 UMCs. In aggregate, our findings support the possibility that oestrogens play a direct and detrimental role in the development of FPAH, and that the risk of FPAH is affected by variations in CYP1B1.

\section{SUPPORT STATEMENT}

Sources of support: National Institutes of Health, National Heart, Lung, and Blood Institute P01 HL72058, K12 RR1 7697 and P01 HL072058 (Bethesda, MD, USA); and the Vanderbilt General Clinical Research Center grant M01 RR 000095 NCRR/NIH (Nashville, TN, USA).

\section{STATEMENT OF INTEREST}

None declared.

\section{ACKNOWLEDGEMENTS}

The authors thank the many patients and families who graciously contributed to this work.

\section{REFERENCES}

1 Loyd JE, Parker B. Francis Lecture. Genetics and gene expression in pulmonary hypertension. Chest 2002; 121: Suppl. 3, 46S-50S.

2 Runo JR, Loyd JE. Primary pulmonary hypertension. Lancet 2003; 361: 1533-1544.

3 Yamaki S, Wagenvoort CA. Comparison of primary plexogenic arteriopathy in adults and children. A morphometric study in 40 patients. Br Heart J 1985; 54: 428-434.

4 Farhat MY, Vargas R, Dingaan B, et al. In vitro effect of oestradiol on thymidine uptake in pulmonary vascular smooth muscle cell: role of the endothelium. Br J Pharmacol 1992; 107: 679-683.

5 West J, Cogan J, Geraci M, et al. Gene expression in BMPR2 mutation carriers with and without evidence of pulmonary arterial hypertension suggests pathways relevant to disease penetrance. BMC Med Genomics 2008; 1: 45.

$6 \mathrm{Kim} \mathrm{JH}$, Sherman ME, Curriero FC, et al. Expression of cytochromes P450 1A1 and 1B1 in human lung from smokers, non-smokers, and ex-smokers. Toxicol Appl Pharmacol 2004; 199: 210-219. 
7 Spivack SD, Hurteau GJ, Reilly AA, et al. CYP1B1 expression in human lung. Drug Metab Dispos 2001; 29: 916-922.

8 Roos PH, Bolt HM. Cytochrome P450 interactions in human cancers: new aspects considering CYP1B1. Expert Opin Drug Metab Toxicol 2005; 1: 187-202.

9 Sorensen M, Autrup H, Tjonneland A, et al. Genetic polymorphisms in CYP1B1, GSTA1, NQO1 and NAT2 and the risk of lung cancer. Cancer Lett 2005; 221: 185-190.

10 Wenzlaff AS, Cote ML, Bock $\mathrm{CH}$, et al. CYP1A1 and CYP1B1 polymorphisms and risk of lung cancer among never smokers: a population-based study. Carcinogenesis 2005; 26: 2207-2212.

11 Yoon KA, Kim JH, Gil HJ, et al. CYP1B1, CYP1A1, MPO, and GSTP1 polymorphisms and lung cancer risk in never-smoking Korean women. Lung Cancer 2008; 60: 40-46.

12 Bugano DD, Conforti-Froes N, Yamaguchi NH, et al. Genetic polymorphisms, the metabolism of estrogens and breast cancer: a review. Eur J Gynaecol Oncol 2008; 29: 313-320.

13 Diergaarde B, Potter JD, Jupe ER, et al. Polymorphisms in genes involved in sex hormone metabolism, estrogen plus progestin hormone therapy use, and risk of postmenopausal breast cancer. Cancer Epidemiol Biomarkers Prev 2008; 17: 1751-1759.

14 Van Emburgh BO, Hu JJ, Levine EA, et al. Polymorphisms in CYP1B1, GSTM1, GSTT1 and GSTP1, and susceptibility to breast cancer. Oncol Rep 2008; 19: 1311-1321.

15 Tsuchiya Y, Nakajima M, Yokoi T. Cytochrome P450-mediated metabolism of estrogens and its regulation in human. Cancer Lett 2005; 227: 115-124.

16 Yager JD, Davidson NE. Estrogen carcinogenesis in breast cancer. N Engl J Med 2006; 354: 270-282.

17 Nebert DW. Elevated estrogen $16 \alpha$-hydroxylase activity: is this a genotoxic or nongenotoxic biomarker in human breast cancer risk? J Natl Cancer Inst 1993; 85: 1888-1891.

18 Muti P, Bradlow HL, Micheli A, et al. Estrogen metabolism and risk of breast cancer: a prospective study of the 2:16 $\alpha$-hydroxyestrone ratio in premenopausal and postmenopausal women. Epidemiology 2000; 11: 635-640.

19 Agundez JA. Cytochrome P450 gene polymorphism and cancer. Curr Drug Metab 2004; 5: 211-224.

20 Simonneau G, Galie N, Rubin LJ, et al. Clinical classification of pulmonary hypertension. J Am Coll Cardiol 2004; 43: Suppl. S, 5S-12S.

21 Cogan JD, Pauciulo MW, Batchman AP, et al. High frequency of BMPR2 exonic deletions/duplications in familial pulmonary arterial hypertension. Am J Respir Crit Care Med 2006; 174: 590-598.

22 Cogan JD, Vnencak-Jones CL, Phillips JA 3rd, et al. Gross BMPR2 gene rearrangements constitute a new cause for primary pulmonary hypertension. Genet Med 2005; 7: 169-174.

23 Machado RD, Aldred MA, James V, et al. Mutations of the TGFbeta type II receptor BMPR2 in pulmonary arterial hypertension. Hum Mutat 2006; 27: 121-132.

24 Bradlow HL, Sepkovic DW, Klug T, et al. Application of an improved ELISA assay to the analysis of urinary estrogen metabolites. Steroids 1998; 63: 406-413.

25 Falk RT, Rossi SC, Fears TR, et al. A new ELISA kit for measuring urinary 2-hydroxyestrone, $16 \alpha$-hydroxyestrone, and their ratio: reproducibility, validity, and assay performance after freeze-thaw cycling and preservation by boric acid. Cancer Epidemiol Biomarkers Prev 2000; 9: 81-87.
26 Klug TL, Bradlow HL, Sepkovic DW. Monoclonal antibody-based enzyme immunoassay for simultaneous quantitation of 2- and 16 $\alpha$-hydroxyestrone in urine. Steroids 1994; 59: 648-655.

27 Humbert M. Update in pulmonary arterial hypertension 2007. Am J Respir Crit Care Med 2008; 177: 574-579.

28 Lahm T, Crisostomo PR, Markel TA, et al. Exogenous estrogen rapidly attenuates pulmonary artery vasoreactivity and acute hypoxic pulmonary vasoconstriction. Shock 2008; 30: 660-667.

29 Morrell NW. Pulmonary hypertension due to BMPR2 mutation: a new paradigm for tissue remodeling? Proc Am Thorac Soc 2006; 3: 680-686.

30 Gonzalez FJ. The molecular biology of cytochrome P450s. Pharmacol Rev 1988; 40: 243-288.

31 Sissung TM, Price DK, Sparreboom A, et al. Pharmacogenetics and regulation of human cytochrome P450 1B1: implications in hormone-mediated tumor metabolism and a novel target for therapeutic intervention. Mol Cancer Res 2006; 4: 135-150.

32 Belous AR, Hachey DL, Dawling S, et al. Cytochrome P450 1B1mediated estrogen metabolism results in estrogen-deoxyribonucleoside adduct formation. Cancer Res 2007; 67: 812-817.

33 Eliassen AH, Missmer SA, Tworoger SS, et al. Circulating 2hydroxy- and 16 $\alpha$-hydroxy estrone levels and risk of breast cancer among postmenopausal women. Cancer Epidemiol Biomarkers Prev 2008; 17: 2029-2035.

34 Kaaks R, Rinaldi S, Key TJ, et al. Postmenopausal serum androgens, oestrogens and breast cancer risk: the European prospective investigation into cancer and nutrition. Endocr Relat Cancer 2005; 12: 1071-1082.

35 Missmer SA, Eliassen AH, Barbieri RL, et al. Endogenous estrogen, androgen, and progesterone concentrations and breast cancer risk among postmenopausal women. J Natl Cancer Inst 2004; 96: 1856-1865.

36 Muti $\mathrm{P}$, Westerlind $\mathrm{K}, \mathrm{Wu} \mathrm{T}$, et al. Urinary estrogen metabolites and prostate cancer: a case-control study in the United States. Cancer Causes Control 2002; 13: 947-955.

37 Bradlow HL, Hershcopf RJ, Martucci CP, et al. Estradiol $16 \alpha$ hydroxylation in the mouse correlates with mammary tumor incidence and presence of murine mammary tumor virus: a possible model for the hormonal etiology of breast cancer in humans. Proc Natl Acad Sci USA 1985; 82: 6295-6299.

38 Tofovic SP, Zhang X, Jackson EK, et al. 2-Methoxyestradiol mediates the protective effects of estradiol in monocrotaline-induced pulmonary hypertension. Vascul Pharmacol 2006; 45: 358-367.

39 Tofovic SP, Zhang $\mathrm{X}, \mathrm{Zhu} \mathrm{H}$, et al. 2-Ethoxyestradiol is antimitogenic and attenuates monocrotaline-induced pulmonary hypertension and vascular remodeling. Vascul Pharmacol 2008; 48: 174-183.

40 Greenlee H, Chen Y, Kabat GC, et al. Variants in estrogen metabolism and biosynthesis genes and urinary estrogen metabolites in women with a family history of breast cancer. Breast Cancer Res Treat 2007; 102: 111-117.

41 Shih MC, Whittemore AS. Tests for genetic association using family data. Genet Epidemiol 2002; 22: 128-145.

42 Roberts KE, Fallon MB, Krowka MJ, et al. Genetic risk factors for portopulmonary hypertension in patients with advanced liver disease. Am J Respir Crit Care Med 2009; 179: 835-842. 